\title{
Notes on the behaviour of trajectories of polynomial dynamic systems
}

\author{
Irina Andreeva ${ }^{1, *}$ \\ ${ }^{1}$ Peter the Great St. Petersburg Polytechnic University, 29 Polytechnicheskaya, St. Petersburg 195251, \\ Russia
}

\begin{abstract}
Dynamic systems play a key role in various directions of modern science and engineering, such as the mathematical modeling of physical processes, the broad spectrum of complicated and pressing problems of civil engineering, for example, in the analysis of seismic stability of constructions and buildings, in the fundamental studies of computing and producing systems, of biological and sociological events. A researcher uses a dynamic system as a mathematical apparatus to study some phenomena and conditions, under which any statistical events are not important and may be disregarded. The main task of the theory of dynamic systems is to study curves, which differential equations of this system define. During such a research, firstly we need to split a dynamic system's phase space into trajectories. Secondly, we investigate a limit behavior of trajectories. This research stage is to reveal equilibrium positions and make their classification. Also, here we find and investigate sinks and sources of the system's phase flow. As a result, we obtain a full set of phase portraits, possible for a taken family of differential dynamic systems, which describe a behavior of some process. Namely polynomial dynamic systems often play a role of practical mathematical models hence their investigation has significant interest. This paper represents the original study of a broad family of differential dynamic systems having reciprocal polynomial right parts, and describes especially developed research methods, useful for a wide spectrum of applications.
\end{abstract}

\section{Introduction}

Contemporary high technologies and broad spectrum of branches of science and knowledge, such as a mathematical modeling of processes in the fields of modern physics and geophysics [17, 21-23], or in urgent key problems of civil engineering, i.e., in the analysis of seismic stability of constructions and buildings [27-30], as well as in some other topics of architectural and construction analysis; in practical and theoretical studies and development of computing and producing systems [20,21], of theoretical and strategical biological problems and phenomena, and of economical and sociological processes and

\footnotetext{
*Corresponding author: $\underline{\text { irandr@inbox.ru }}$
} 
sometimes unexpected events (such as functioning of a state as a complicated system [17, 18]; mathematical modeling of peaceful trade or even military interactions between different states [16, 19]), all these different scientific and engineering tasks cannot be solved without dynamic systems' consideration and research. Dynamic systems have in them a key significance. A proper dynamic system is used as a mathematical apparatus in case of study of those phenomena or situations, when statistical events (they are often called fluctuations) may not be taken into consideration and can be omitted [31].

The dynamic systems' qualitative mathematical theory helps us to find and define curves, which are described by the considered differential equations. Working over this task, firstly it is needed to split a phase space into determined trajectories. Secondly, a researcher has to outline their limit behavior [10, 11, 24-26] with the goal to find equilibrium positions, to proceed their investigation separately and obtain the detailed classification of them. At this step it becomes necessary to describe sinks and sources of a phase flow.

The main items of any dynamic system investigation are to be the follows.

A) To answer a question concerning a character of equilibrium states, i.e. their stability, or a property of a studied dynamic system to keep closely to its equilibrium state despite of (small enough) changes in the initial data. Or to persist on a given manifold during some considerably prolongated period of time.

B) To clarify the issue of a roughness, i.e. this system's ability to keep its features despite of (restricted enough) modifications of a model, which a system is used to portray. Rough systems keep stable their qualitative features of motion despite of arbitrary restricted variations of parameters.

The given paper is dedicated to a presentation of the original investigation, the especially developed research methods used in it, and precise strict conclusions of this mathematical consideration, proceeded in the field of the qualitative theory of dynamic systems and ordinary differential equations. Those research methods are effective, new and original ones, thus they will appear helpful for further steps in the studies of different applied polynomial dynamic systems.

The beginnings of the (ordinary) differential equations' qualitative theory were established by the great encyclopedist Jules H. Poincare (1854 - 1912), who taught us, that every - considered on an extended real plane $\bar{R}_{x, y}^{2}$ - second ordered polynomial normal autonomous differential system may be completely qualitatively studied [1, 12-14].

Later the quadratic systems' features were outlined, together with a series of other special kinds of polynomial dynamic systems [2-4].

Here we investigate a special, but considerably extended class (which may be called also a family) of polynomial systems taken on an arithmetical phase plane $x, y$

$$
\frac{d x}{d t}=X(x, y), \quad \frac{d y}{d t}=Y(x, y)
$$

where $X(x, y), Y(x, y)$ be reciprocal polynomials, $X$ is taken as a cubic form, while $Y$ is considered as a square form, with the restrictions (but without detracting from the generality of reasoning

$$
X(0,1)>0, \quad Y(0,1)>0
$$

The J.H. Poincare's method of sequential mappings (via the two Poincare's transformations) leads to the achievement of the set mathematical goals and obtaining of the full qualitative pattern of trajectories with depicting in a Poincare disk of all possible for the systems (1) different (under the topological understanding) phase portraits. 
This investigating plan includes the items:

1) We display the phase plane $\bar{R}_{x, y}^{2}$ of the investigated differential system on the Poincare sphere $\sum$ through a central mapping (from the center $(0,0,1)$ of the Poincare sphere $\sum$

$$
X^{2}+Y^{2}+Z^{2}=1
$$

(the diametrically opposite points on the Poincare sphere $\sum$ are to be considered identified).

2) We map on the Poincare disk $\bar{\Omega}$ (enclosed), through the orthogonal mapping, the lower enclosed semi sphere of the Poincare sphere $\sum$

$$
x^{2}+y^{2} \leq 1
$$

(the diametrically opposite points situated at its boundary $\Gamma$ are also considered to be identified).

\section{Key notions}

$\varphi(t, p), p=(x, y)-\mathrm{a}$ fixed point $:=$ be a motion with the initial data $(0, p)$. That means a solution of some system belonging to the family defined as (1).

$L_{p}: \varphi=\varphi(t, p), t \in I_{\max }$, - be a dynamic system's (1) trajectory corresponding to a motion $\varphi(t, p)$.

$L_{p}^{+(-)}:=+(-)$- be a semi trajectory of a those dynamic system's (1) trajectory $L_{p}$.

$O$-curve of a dynamic system $:=$ be a semi trajectory $L_{p}^{s}(p \neq O, s \in\{+,-\})$ adjoining to a point $O$ subjecting to the requirement that $s t \rightarrow+\infty$.

$O^{+(-)}$- curve of a dynamic system $:=$be the system's $O$-curve $L_{p}^{+(-)}$.

$O_{+(-)^{-}}$curve of a dynamic system := be the system's $O$-curve, adjoining to a point $O$ while coming out of the domain $x>0 \quad$ ( coming out of the domain $x<0$ alternatively).

$T O$-curve of a system $:=$ be the dynamic system's $O$-curve, which touches some ray in the $O$ point under the condition of being supplemented with the point $O$.

A nodal bundle of $N O$-curves of a dynamic system:= be the continuous open family of the taken system's (1) TO-curves $L_{p}^{s}$, where $s \in\{+,-\}$ be a fixed index, $p \in \Lambda, \Lambda$ - be a simple open arc, for which $L_{p}^{s} \cap \Lambda=\{p\}$.

A saddle bundle of $S O$-curves of a system (or a separatrix of a point $O$ ) $:=$ be a fixed $T O$ -curve, belonging to no one bundle of $N O$-curves of a system.

$H, P, E-$ be the $O$-sectors: a hyperbolic sector, a parabolic sector, and an elliptical sector of a dynamic system.

A T-type of a singular point $O$ of a dynamic system := be a word $A_{O}$ including letters $N$, $S$, which is used to describe an order of bundles $N, S$ of $O$-curves (when we going counter clockwise around the point $O$, beginning from some of them (that means to move in the socalled «+»-direction). In the expanded form we'll call the T-type of a singular point $O$ as its topological type.

In addition for research purposes we portray a topological type of a singular point $O$ via a word $B_{O}$, composed of letters $E, H, P$, helping to draw the possible circular sequential order of $O$-sectors $E, H, P$ around the (finite singular) point $O$ bypassed as well in the «+»direction (starting with some of them likewise).

The following special polynomials associated with the system (1) will be necessary: 


$$
\begin{gathered}
P(u):=X(1, u) \equiv p_{0}+p_{1} u+p_{2} u^{2}+p_{3} u^{3} \\
Q(u):=Y(1, u) \equiv a+b u+c u^{2}
\end{gathered}
$$

All dynamic systems (1) appear to have the properties:

A) $A_{O}$ or $B_{O}$, the two forms of representation of a T-type of a point $O$, easily may be transformed one into another.

B) For the special polynomial $Q(u)$ its real roots represent in fact angular coefficients of the isoclines of a zero.

C) For the special polynomial $P(u)$ its real roots represent in fact angular coefficients of the isoclines of infinity.

D) Let's agree to number those real roots of polynomials $P(u), Q(u)$ (see items above) in ascending order always.

The important note.

Since the polynomials in right parts of equations for the systems (1) are considered to be reciprocal ones, all roots of the special polynomial $P(u)$ are different from roots of the special polynomial $Q(u)$.

Investigation and description of the possible topological types ( $\mathrm{T}$ - types) appearing for a singular point $O(0,0)$ together with the existing infinitely remote singular points of dynamic systems (1) are represented in papers [5-9].

\section{Hierarchical subfamilies. Polynomial reciprocal right parts including 3 and 1 multipliers}

Proceeding through the detailed study of the whole extended systems (1) class or family, it becomes vital to divide it into subfamilies using some natural features of them. There appeared to exist up to four hierarchical levels of such subfamilies of dynamic systems under investigation. The upper level of their hierarchy is defined with the numbers of different multipliers in their decompositions into polynomials of lower orders [32, 33].

Here we describe a subfamily

$$
\begin{gathered}
\frac{d x}{d t}=p_{3}\left(y-u_{1} x\right)\left(y-u_{2} x\right)\left(y-u_{3} x\right), \frac{d y}{d t}=c\left(y-q_{1} x\right)^{2} \\
p_{3}>0, c>0, u_{1}<u_{2}<u_{3}, q(\in R) \neq u_{i, i} i=\overline{1,3}
\end{gathered}
$$

The following research suggests several common for different upper level subfamilies steps. Let's subdivide the subfamily (6) into the sub-subfamilies numbered by $r=\overline{1,4}$.

Let's call the $R S P Q$ the sequence of roots of polynomials $P(u)$ and $Q(u)$ for this subfamily, which are written in the ascending order.

Every of those sub-subfamilies will be a totality of systems with an $R S P Q$ number $r$, where $r$ is its number in the list of possible $R S P Q$ s.

$$
\begin{array}{ll}
\text { 1. } & u_{1}, u_{2}, u_{3}, q, \\
\text { 2. } & u_{1}, u_{2}, q, u_{3}, \\
\text { 3. } & u_{1}, q, u_{2}, u_{3}, \\
\text { 4. } & q, u_{1}, u_{2}, u_{3} .
\end{array}
$$

Using for the system (6) a so-called double change of variables (DC): $(t, y) \rightarrow(-t,-y)$, we can see, that this change allows to transform subfamilies having numbers $r=1,2,3,4$, into families numbered with $r=4,3,2,1$ correspondingly (as well as backwards). This note means, that subfamilies of systems (6) numbered with 1 and 2 are not connected one to 
another via the double change transformation, and subfamilies numbered with 3 and 4 are not related also; but the subfamily number 3 is "mutually inversed" via the DCtransformation with regards to the family number 2 , as well as subfamily number 4 is "mutually inversed" to the family number 1 (correspondingly). This is easy to obtain from the analysis of their $R S P Q$ sequences [5 -9, 11].

Further we stick to the common research program that provides the following steps.

1) We investigate subfamilies of systems, $r=1,2$, i.e.:

1. We additionally split the taken subfamily into sub-subfamilies, belonging to a deeper hierarchical level, and numbered with $s[5,6], s=\overline{1,9}$, and find out the topodynamical types (TD-types) of singular points of these systems [5-10].

2. We depict for the systems of a fixed sub-subfamily $\forall s=\overline{1,9}$ the so-called "offroad map" [5 -7]. This "off-road map" is used further with the aim to investigate separatrices and theirs limit behavior, as well as it lets us to reveal for all separatrices their mutual pattern of the arrangement in the Poincare disk $\Omega$.

3. We obtain and describe all existing and possible (topologically different) phase portraits in the Poincare disk for systems correspond to the equations (6).

2) After that we study the subfamilies correspond to $r=3,4$, using the double change transformation of results earlier received for subfamilies correspond to $r=2,1$, and portray existing phase portraits for subfamilies 3 and 4 .

Conclusions for this paragraph are the follows. For sub-subfamilies of systems (6) with numbers $1,2,3$ and 4 will exist $15+11+11+15=52$ topologically different (types) of phase portraits in a Poincare disk $\bar{\Omega}[32,33]$.

In the way outlined before we can investigate all other possible (and depending on the pattern and sequences of different roots including in the RSPQs) hierarchical subfamilies belong to the upper level, gradually sinking to deeper levels of detail [15, 24-26].

\section{Recommendations}

Paper is proposed to be useful for students, postgraduates, engineers and scientific researchers interested in the fields of the qualitative theory of ordinary differential equations, dynamic systems and their applications to the broad spectrum of branches of contemporary science and engineering, such as the mathematical modeling of processes which are significant in important problems of civil engineering, i.e., in the analysis of seismic stability [27-30] and others; studies of producing and computing systems [20, 21], research of biological, sociological [31] and economical events and phenomena [16-19, 22, 23].

The author is immensely grateful to Professor Dr. Alexey Fedorovich Andreev from St. Petersburg State University (1923 - 2017), for His greatly valued contribution into this research topic.

Also, the author is deeply grateful to Professor Dr. Valeriy Konstantinovich Zakharov, Lomonosov Moscow State University, for constant constructive and fruitful mathematical and physical scientific discussions.

\section{References}

1. A. Andronov, E. Leontovich, I. Gordon, A. Maier, Qualitative theory of second-order dynamic systems (New York: Wiley, 1973)

2. A. Andreev, I. Andreeva, Differential Equations, 33(5), 702-703 (1997) 
3. A. Andreev, I. Andreeva, 2007 Vestnik St. Petersburg University, Ser ,1 Mathematics, Mechanics, Astronomy, 2, 11-16 (2007)

4. A. Andreev, I. Andreeva, L. Detchenya, T. Makovetskaya, A. Sadovskii, Differential Equations, 53(8), 1003-1008 (2017)

5. A. Andreev, I. Andreeva, Differential Equations and Control, 4,17-26 (2007)

6. A. Andreev, I. Andreeva, Differential Equations and Control, 1, 1-13 (2008)

7. A. Andreev, I. Andreeva, Differential Equations and Control, 3, 39- 49 (2008)

8. A. Andreev, I. Andreeva, Differential Equations and Control, 4, 181-213 (2009)

9. A. Andreev, I. Andreeva, Differential Equations and Control, 4, 6-17 (2010)

10. A. Andreev, I. Andreeva, Vibroengineering Procedia, 15, 88-93 (2017)

11. A. Andreev, I. Andreeva, IOP Journal of Physics: Conference Series, 1141, 012114 (2018)

12. H. Poincare, On curves defined by differential equations (Moscow, OGIS, 1947)

13. H. Poincare, Les methodes nouvelles de la mecanique celeste (Paris, 1892 - 99)

14. H. Poincare, Proc. Royal Soc., London, 91, 5 - 16 (1915)

15. I. Andreeva, T. Efimova, IOP Journal of Physics: Conference Series, 1236, 012053 (2019)

16. V. Zakharov, Vibroengineering PROCEDIA, 25, 143 - 150 (2019)

17. V. Zakharov, O. Kuzenkov, Modeling and data analysis, 1, 55-75 (2011)

18. V. Zakharov, D. Kapitanov, O. Kuzenkov, Modeling and data analysis, 1, 4-31 (2014)

19. V. Zakharov, Abstracts of 26 International conference Mathematics Computer Education (28 January-2 February 2019), Russia, Pushchino: Pushchino Center of Biological Investigations, 238 (2019)

20. V. Zakharov, Current research trends of the XXI century: Theory and Practice, 5, 10 (36), 219-222 (2017)

21. V. Zakharov, Modeling and data analysis, 1 (1), 21-36 (2016)

22. O. Aksenova, I. Khalidov, Rarefied Gas Dynamics AIP Conference Proceeding, 1786, American Institute of Physics, Melville, NY, 1000091-1000097 (2016)

23. O. Aksenova, I. Khalidov, Rarefied Gas Dynamics AIP Conference Proceedings, 1786, American Institute of Physics, Melville, NY, 1000071- 1000078 (2016)

24. A. Andreev, I. Andreeva, Vestnik RAEN, 4 (17), 11-15 (2017)

25. A. Andreev, I. Andreeva, Vestnik RAEN, 4 (18), 8-18 (2018)

26. A. Andreev, I. Andreeva, Vestnik RAEN, 4 (19), (2019)

27. K. Fahmi, E. Kolosov, M. Fattah, Journal of Engineering and Applied Sciences, 14(4), 1162-1168 (2019)

28. E. Kolosov, K. Agishev, N. Kolosova, MATEC Web of Conferences, 107, 00032, (2017)

29. N. Kolosova, E. Kolosov, K. Agishev, MATEC Web of Conference, 107, 00065 (2017)

30. D. Rashid, E. Kolosov, N. Kolosova, T. Soldatenko, Advances and Trends in Engineering Sciences and Technologie, II, Proceedings of the 2nd International Conference on Engineering Sciences and Technologies: ESaT (2016) 
31. V. Zakharov, IOP Journal of Physics: Conference Series, 1391, 012040 (2019)

32. I. Andreeva, IOP Journal of Physics: Conference Series, 1391, 012039 (2019)

33. I. Andreeva, T. Efimova, IOP Journal of Physics: Conference Series, 1425, 012040 (2019) 